\title{
Mechanism and Reconstitution In Vitro of Germ Cell Development in Mammals
}

\author{
KaZUKi KuRimoto ${ }^{1,2}$ and Mitinori Saitou ${ }^{1,2,3,4}$ \\ ${ }^{1}$ Department of Anatomy and Cell Biology, Graduate School of Medicine, Kyoto University, \\ Yoshida-Konoe-cho, Sakyo-ku, Kyoto 606-8501, Japan \\ 2JST, ERATO, Yoshida-Konoe-cho, Sakyo-ku, Kyoto 606-8501, Japan \\ ${ }^{3}$ Center for iPS Cell Research and Application, Kyoto University, Shogoin, Sakyo-ku, \\ Kyoto 606-8507, Japan \\ ${ }^{4}$ Institute for Integrated Cell-Material Sciences, Kyoto University, Yoshida-Ushinomiya-cho, \\ Sakyo-ku, Kyoto 606-8501, Japan \\ Correspondence: saitou@anat2.med.kyoto-u.ac.jp; kurimoto@anat2.med.kyoto-u.ac.jp
}

\begin{abstract}
The germ cell lineage creates new individuals, perpetuating/diversifying the genetic and epigenetic information across generations. Based on the knowledge obtained through investigations into the mechanisms of germ cell specification and development in mice, we have succeeded in precisely reconstituting the specification and subsequent development of germ cells in culture in both males and females: Embryonic stem cells (ESCs)/induced pluripotent stem cells (iPSCs) are induced into epiblast-like cells (EpiLCs) and then into primordial germ cell-like cells (PGCLCs), which robustly contribute to spermatogenesis and oogenesis and to fertile offspring. This in vitro mouse PGC specification/development system has led to the elucidation of signaling, transcriptional, and epigenetic regulation during germ cell development in a detailed fashion. More recently, based on this system, we and others have demonstrated the induction of human PGCLCs from human ESCs/iPSCs, creating an opportunity for understanding the mechanism of human germ cell development in vitro.
\end{abstract}

Germ cells are the only cell type that generates the full developmental potency to give rise to the next generations (totipotency). To carry out this fundamental role, primordial germ cells (PGCs), the precursors for the spermatozoa and the oocytes, undergo a number of unique events prior to their differentiation into spermatogonial stem cells (SSCs) or oocytes, which include repression of somatic programs, (re)acquisition of potential pluripotency, and reprogramming of epigenome information, for which, in the last decade, we and other groups have clarified at least a part of the molecular mechanisms (Surani and Hajkova 2010; Saitou and Yamaji 2012; Saitou et al. 2012). Based on these studies, we have successfully reconstituted the mouse germ cell specification pathway in culture: The embryonic stem cells (ESCs)/ induced pluripotent stem cells (iPSCs) are induced into epiblast-like cells (EpiLCs), which are in turn induced into PGC-like cells (PGCLCs) with robust capacities both for spermatogenesis and oogenesis (Hayashi et al. 2011, 2012). The in vitro PGC specification/development system has been a basis for elucidation of the signaling, transcriptional, and epigenetic regulation associated with PGC specification and development (Aramaki et al. 2013; Nakaki et al. 2013; Kurimoto et al. 2015). Such knowledge would have been difficult to obtain using the in vivo materials, and thus the in vitro system will continue to be applied to answer many remaining questions in germ cell biology. More recently, based on this mouse system, we and others have achieved the induction of human PGCLCs from human pluripotent stem cells (hPSCs) (Irie et al. 2015; Sasaki et al. 2015), which creates an unprecedented opportunity for understanding the mechanism for human germ cell development. In this manuscript, we summarize recent advances in elucidation of the mechanisms underlying germ cell development in both mice and humans, as well as in their reconstitution in vitro.

\section{OUTLINE AND KEY MOLECULAR EVENTS OF PGC DEVELOPMENT IN MICE}

The mammalian germline is set aside from somatic lineages in early postimplantation embryos. Mouse primordial germ cells (mPGCs) emerge from the epiblasts, a pluripotent epithelium that gives rise to all embryonic tissues. Upon the onset of gastrulation at around embryonic day (E) 6.25, the most proximal posterior epiblast cells receive a high dose of bone morphogenic protein 4 (BMP4) signaling from the adjoining extraembryonic ectoderm and are induced into mPGCs, which form a cluster of alkaline phosphatase-positive cells within the extraembryonic mesoderm at $\sim$ E7.25 (Fig. 1; Ginsburg et al. 1990; Lawson et al. 1999; Saitou et al. 2002). PR domain-containing 1 (Prdm 1; also referred to as Blimp 1) is expressed in the precursors of mPGCs, and was identified as a critical determinant of the germ cell fate (Ohinata et al. 2005; Vincent et al. 2005). Molecular techniques, including single cell microarray analysis 




Figure 1. Schematic representation of the character and key molecular events in specification of human and mouse germ cell fates. The peri-implantation development in primates and mice is represented with a schematic drawing made with reference to Luckett (1978) and Kaufman (1992). The inner cell mass, ectoderm, mesoderm, and endoderm are indicated with pale blue, purple, yellow, and pink, respectively. Primordial germ cells (PGCs) are represented with green circles. The cells derived from PSCs in vitro are represented with circles. PSCs, pluripotent stem cells; EpiLCs, epiblast-like cells; PGCLCs, primordial germ cell-like cells; TF, transcription factor; iMeLCs, incipient mesoderm-like cells.

(Kurimoto et al. 2006), have demonstrated that BLIMP1 robustly represses the somatic mesodermal program transiently activated upon mPGC specification (the program for "pattern specification," "embryonic morphogenesis," and "gastrulation," etc.), allowing mPGCs to escape from the mortal somatic cell fate, and is thus essential for the establishment of all of the subsequent events in PGCs (Ohinata et al. 2005; Yabuta et al. 2006; Kurimoto et al. 2008).

Concomitant with repression of the somatic program, mPGCs regain a key transcription network for pluripotency, including Oct3/4 (Pou5f1), Sox2, and Nanog, and indeed, mPGCs can dedifferentiate into PSCs under particular culture conditions (embryonic germ cells) (Matsui et al. 1992). Therefore, although mPGCs themselves are not pluripotent, they (re)acquire pluripotency in a hidden form (potential pluripotency).
After specification, at $\sim$ E7.25, mPGCs start to migrate into endoderm epithelium of hindgut toward future gonads, which will be formed in later developmental stages (at $\sim$ E10.5). The migratory mPGCs appear to arrest the cell cycle and commence epigenome reprogramming, including genome-wide DNA demethylation and reorganization of repressive histone modifications (Seki et al. 2005, 2007; Saitou et al. 2012). Defects in the epigenetic regulation lead to a variety of deficiencies in germ cell development, including loss of germ cells, impaired development of embryos and offspring, and inherited epigenetic abnormalities (Heard and Martienssen 2014), and therefore, epigenome reprogramming is considered to be a critical process in allowing mPGCs to establish a totipotent epigenome for the next generations.

Another PR domain-containing factor, Prdm14, was identified as a critical requirement for mPGC specifica- 
tion with specific expression in mPGCs in response to BMP4. Prdm14 deficiency led to ablation in the (re)acquisition of potential pluripotency and epigenome reprogramming (Yamaji et al. 2008). More recently, Prdm14 was shown to play a critical role for the naïve pluripotency of mouse embryonic stem cells (mESCs) by antagonizing fibroblast growth factor (FGF) signaling and repressing de novo DNA methylation machinery (Ma et al. 2011; Yamaji et al. 2013). Therefore, among the key molecular events for mPGC specification, these studies showed that epigenome reprogramming and (re)acquisition of potential pluripotency are regulated by $\operatorname{Prdm} 14$, whereas all of the key PGC events including repression of somatic programs are governed by Blimpl (Fig. 1).

The migratory mPGCs exit from the hindgut, pass through the dorsal mesentery with highly active proliferation, and then enter the embryonic gonads, where they start sex-specific development (for males, mitotic arrest; for females, prophase I of meiosis). It is important to note that, prior to sex-specific development, mPGCs almost completely erase the genome-wide DNA methylation, including parental imprints, with the acquisition of unique histone modifications (Seisenberger et al. 2012; Lee et al. 2014).

\section{RECONSTITUTION IN CULTURE OF THE MOUSE GERM CELL SPECIFICATION PATHWAY}

The reconstruction of germ cell development in vitro has been considered an important challenge for the precise understanding of the molecular mechanisms of germ cell development and for the regeneration of reproductive capacities in a given species. Accordingly, in vitro differentiation of germ cell-like cells from ESCs has been attempted by exploiting random differentiation in embryoid bodies, but with limited success (Daley 2007). Ohinata et al. (2009) clarified a signaling principle for mPGC specification from the epiblasts and demonstrated that isolated mouse epiblasts are efficiently induced into Blimp 1-positive and Prdm14-positive PGC-like cells in floating aggregates under a defined condition with stimulation by cytokines including BMP4, and that, remarkably, these cells successfully contribute to spermatogenesis upon transplantation into the testes of neonatal mice lacking endogenous germ cells ( $W / W^{v}$ mice). Therefore, the generation of an in vitro counterpart of the epiblasts was reasoned to be an essential step for in vitro induction of the germ cell fate from mPSCs. On the other hand, PSCs derived from the epiblasts, known as epiblast-stem cells (EpiSCs) (Brons et al. 2007; Tesar et al. 2007), exhibited a subpopulation expressing germ cell markers, and this population was increased by stimulation with BMP4, but only with limited efficiency (Hayashi and Surani 2009). Considering that the competence of the epiblasts to produce mPGCs is a transient property (E5.5-E6.25) (Ohinata et al. 2009), the low efficiency of EpiSCs for the germ cell fate was attributed to their becoming incompatible for efficient PGC induction during culture.
Given these findings, Hayashi et al. developed a strategy to induce pregastrulating EpiLCs from mPSCs in a ground-state pluripotency (Ying et al. 2008) by stimulation with activin A and basic fibroblast growth factor. Similar to the epiblasts at around E5.5-E6.25, EpiLCs exhibited an efficient competence to generate mPGC-like cells (mPGCLCs) in a floating culture in response to BMP4, and mPGCLCs, upon transplantation into the testes of neonatal $W / W^{\nu}$ mice, showed a robust capability to produce spermatozoa, which contributed to healthy offspring (Hayashi et al. 2011). The competence of EpiLCs for mPGCLC generation reached an optimal level at around day 2 of EpiLC induction, and thereafter decreased, recapitulating the transient competence of the epiblasts to form PGC-like cells.

The transcriptomes of EpiLCs and mPGCLCs at day 6 of induction (d6 mPGCLCs) were very similar to those of E5.75 epiblast and E9.5 migratory mPGCs, respectively. The induction pathway of MPGCLCs well recapitulated that of mPGC specification in vivo; Blimp1-positive mPGCLCs transiently activated a mesoderm-like expression profile at day 2 of induction and robustly repressed it by day 6. d6 mPGCLCs did not show expression of late mPGC genes such as Ddx4 and Piwil2. Importantly, this strategy was also valid for females: Female mPGCLCs were induced from female mPSCs through EpiLCs, and when aggregated with somatic cells of female embryonic gonads (the reconstituted ovaries), female mPGCLCs exhibited further differentiation to acquire the properties of late PGCs (i.e., E12.5 PGCs), and upon a longer culture, progressed into the prophase of the first meiotic division, demonstrating the capacity of mPGCLCs for the meiosis (Hayashi et al. 2012). Remarkably, when the reconstituted ovaries were transplanted under the ovarian bursa of immune-deficient mice, mPGCLCs matured into oocytelike cells at the germinal vesicle stage with the formation of Graafian follicle-like structures, and some of the mPGCLC-derived oocytes, through in vitro maturation and fertilization, contributed to healthy offspring (Hayashi et al. 2012).

These findings demonstrated that the MPGCLC induction from mPSCs via EpiLCs is an in vitro counterpart of germ cell specification and development from the epiblasts in mice, providing a robust foundation for understanding and regulating the mechanism of mammalian germ cell development in both sexes in vitro.

\section{RECONSTITUTION OF MOUSE PGCLCS BY INDUCED TRANSCRIPTION FACTORS}

To examine the intrinsic logic of mPGC specification, Nakaki et al. (2013) explored transcription factors (TFs) sufficient for mPGC specification, and demonstrated an efficient induction $(>80 \%)$ of mPGCLCs by inducible, combinatorial overexpression of three TFs, Blimp1, Prdm14, and Tfap 2c, in EpiLCs (TF-mPGCLCs). Among these three TFs, Prdm 14 was sufficient for induction of the germ cell state by itself, indicating the central role of this factor in conferring the germ cell fate upon EpiLCs. The TF-mPGCLCs reconstituted transcriptome and epi- 
genetic reprogramming in $\mathrm{mPGCs}$, and more notably, they contributed to functional spermatogenesis upon transplantation into the testes of neonatal $W / W^{v}$ mice. Thus, these TFs, particularly $\operatorname{Prdm14}$, are sufficient to create the genetic and epigenetic network for mPGCs (Nakaki et al. 2013).

Upon the induction of the TFs, EpiLCs swiftly acquired the germ cell fate, whereas the mesodermal genes, including T, Hoxal and Hoxb1, were never up-regulated throughout the induction pathway, indicating that the mPGC specification can be carried out without the transient activation of the somatic mesoderm program, raising a question as to the role of the mesoderm program in mPGC specification (see below).

It is of note that the direct induction of the three TFs in mESCs led to the formation of distinct, teratogenic cells incapable of spermatogenesis. This demonstrated a critical requirement of the epigenome background or cellular context of EpiLCs for the TFs to act properly to create the germ cell fate. This study thus established a framework for the transcriptional logic of PGC specification by Blimp1, Prdm14, and Tfap2c, and for exploration of TF-based approaches to elucidate the mechanisms of further germ cell development. Consistent with this study, it was reported independently that the three TFs induce expression of mPGC markers in embryonal carcinoma cells (Magnusdottir et al. 2013).

\section{MOLECULAR BASIS FOR THE COMPETENCE OF THE EPIBLAST FOR THE GERM CELL FATE}

The molecular basis of the competence of the epiblasts and EpiLCs to take on the germ cell fate has been an open question. The expression of WNT3 starts in the pregastrulating epiblast and posterior visceral endoderm (Liu et al. 1999; Rivera-Perez and Magnuson 2005), and WNT3 has been identified as an essential cytokine for the epiblast to express Blimp1 and Prdm 14 in response to BMP4, providing the epiblast cells with the competence for the germ cell fate (Ohinata et al. 2009; Tanaka et al. 2013).

By analyzing $\beta$-Catenin-knockout embryos/mESCs/ EpiLCs and by overexpressing a constitutively active form of $\beta$-Catenin in Wnt3-knockout EpiLCs, Aramaki et al. (2013) demonstrated that the canonical $\beta$-CATENIN-mediated signaling pathway is a key downstream effector of WNT3 for the competence for MPGC specification. The exploration of downstream targets of BMP4 and WNT3/ $\beta$-CATENIN signaling identified $T$ (Brachyury), an evolutionarily conserved TF important for posterior mesoderm formation (Herrmann et al. 1990), as a candidate for conferring the WNT3-mediated competence of the epiblasts. $T$-knockout embryos failed to form mPGCs, whereas overexpression of $T$ in Wnt3knockout EpiLCs robustly activated high-level expression of Blimp1 and Prdm14 without stimulation by WNT3 or BMP4. Indeed, T directly activated the expression of these PGC determinants by binding to their enhancers, indicating that $\mathrm{T}$ plays a primary and sufficient role as the downstream effector of WNT3 signaling.
T induces the PGC determinants in EpiLCs, independently from BMP4 signaling. This raises a question: What is the role of BMP4 in the PGC specification? When EpiLCs were preexposed to WNT3A, overexpression of $T$ did not activate Blimp1 and Prdm14, demonstrating that WNT signaling and/or its downstream effectors inhibit the function of $\mathrm{T}$ in PGC specification, and BMP4 plays a permissive role in allowing $\mathrm{T}$ to activate Blimp1 and Prdm14 (Aramaki et al. 2013). Thus, this study provided the molecular basis of the competence of the epiblast/EpiLCs for the germ cell fate and answered the question of why the mouse germ cell fate is determined at the onset of gastrulation and transiently activates the mesoderm program during the specification. As demonstrated by the TF overexpression experiments (Nakaki et al. 2013), once the mPGC fate is established, the mesodermal program including $T$ is dispensable for the function of mPGCs.

\section{GENOME-WIDE DNA DEMETHYLATION DURING GERM CELL DEVELOPMENT}

One of the key aspects of epigenome reprogramming in mPGCs is the genome-wide DNA demethylation, for which two basic mechanisms had been proposed: one is replication-coupled, passive demethylation, with downregulation of de novo and maintenance DNA methylation machineries upon PGC specification; and the other is enzyme-based active demethylation - that is, deamination of 5-methyl cytidine (5mC) by AID or APOBEC (Guo et al. 2011) and/or oxidation of $5 \mathrm{mC}$ by TET proteins (Kriaucionis and Heintz 2009; Tahiliani et al. 2009), followed by base-excision repair (BER) (Saitou et al. 2012; Lee et al. 2014). The active DNA demethylation was considered a predominant mechanism, based on several sets of findings - namely, the kinetic analysis of DNA demethylation of differentially methylated regions (DMRs) of several imprint genes (Hajkova et al. 2002); the apparent involvement of the cytidine deaminase, Aid, in DNA demethylation in mPGCs (Popp et al. 2010); and the apparent activation of the BER pathway and subsequent dramatic chromatin changes, including loss of linker histone $\mathrm{H} 1$ and of several histone modifications, perhaps through histone replacement, in mPGCs (Hajkova et al. 2010).

Kagiwada et al. (2013) performed quantitative analyses of the genome-wide gene expression profile, cell cycle, nucleus morphology, and DNA methylation dynamics of imprint DMRs. The transcriptome analyses of isolated mPGCs exhibited rapid down-regulation and stable lowlevel expression of Uhrfl (the recruiter of maintenance DNA methyltransferase, DNMT1, into the replication foci) (Sharif et al. 2007) and all of the de novo DNA methyltransferases, supporting the notion that mPGCs consistently possess little maintenance and de novo DNA methylation potential after specification. On the other hand, all of the genes proposed to be involved in active DNA demethylation, except TET1 and $T D G$, were expressed at low or undetectable levels.

mPGCs appeared to exhibit a cell cycle arrest upon migration into the hindgut endoderm at $\sim$ E7.75-E8.75 
and were released from this arrested state upon their exit from the hindgut to the dorsal mesentery at $\sim$ E9.5 (Seki et al. 2007; Kagiwada et al. 2013). Quantitative analyses of the cell cycle using a combination of BrdU incorporation and DNA content showed the restart of rapid and constant proliferation of mPGCs after E9.5, with an average doubling time of $12.6 \mathrm{~h}$, indicating that $\mathrm{mPGCs}$ divide twice per day. In good agreement with the rapid proliferation and the loss of maintenance and de novo DNA methylation activity, bisulfite sequence analysis showed that DNA demethylation was already in progress at E9.5, and the kinetics of imprint erasure in mPGCs did not exceed the demethylation rate that was expected from the replication-coupled passive mechanism, with gene-dependent partial resistances to demethylation. Moreover, Kagiwada et al. (2013) did not detect drastic chromatin changes, such as the loss of linker histone H1 or the loss of several histone modifications, in mPGCs within the embryonic gonads. These findings indicated that, contrary to what had been proposed (Hajkova et al. 2010), the imprint erasure is initiated in early mPGCs and the demethylation can be explained largely by a replication-coupled passive mechanism. Consistent with this report, independent studies for the methylation states of the repetitive elements in mPGCs by highthroughput hairpin bisulfite sequencing demonstrated the prevalence of hemimethylated DNA in mPGCs, indicating that the replication-coupled passive demethylation is a major mechanism for genome-wide DNA demethylation in mPGCs (Seisenberger et al. 2012; Arand et al. 2015). Accordingly, mPGCs lacking TET1, an active DNA demethylase and the only TET protein expressed at a high level in mPGCs, undergo genome-wide DNA demethylation in an apparently normal fashion (Dawlaty et al. 2011). It is, however, important to note that TET1deficient PGCs showed impairment for the DNA demethylation of several specific elements, including DMRs of a few imprinted genes and some genes critical for meiosis, resulting in impaired fertility both in males and females (Yamaguchi et al. 2012, 2013).

\section{CHROMATIN REMODELING DURING GERM CELL DEVELOPMENT}

Concomitant with the progress of genome-wide DNA demethylation, mPGCs reprogram repressive chromatin marks in an apparently genome-wide manner: They down-regulate histone H3 lysine 9 dimethylation (H3K9me2) and simultaneously up-regulate H3K27me3 (Seki et al. 2005), but the scale and specificity of these regulations had been elusive. Kurimoto et al. (2015) clarified the dynamics of the genome-wide remodeling of key histone modifications, H3K9me2 (repression by G9A/ GLP), H3K27me3 (repression by Polycomb repressive complex 2 [PRC2]), H3K4me3 (active promoters), and $\mathrm{H} 3 \mathrm{~K} 27 \mathrm{ac}$ (acetylation) (active enhancers), and the genome-wide binding sites of key TFs, T, and BLIMP1, during in vitro $\mathrm{mPGC}$ specification/development.

The chromatin immunoprecipitation sequencing (ChIP-seq) analysis revealed that, consistent with the ob- servations from immunofluorescence analyses of mPGCs in vivo (Seki et al. 2005, 2007), the repressive mark H3K9me2 exhibited a progressive, genome-wide reduction from day 2 (d2 mPGCLCs, representing mPGC precursors expressing key PGC genes [Blimp1, Prdm14, Tfap 2c] as well as mesodermal genes) to day 6 of induction (d6 mPGCLCs, representing E9.5 mPGCs). Concomitant with the $\mathrm{H} 3 \mathrm{~K} 9 \mathrm{me} 2$ reduction, DAPI-dense heterochromatin and LAMIN B1 around the nuclear periphery in $\mathrm{d} 6 \mathrm{mPGCLCs}$ were diminished, indicating that mPGC(LC)s alter their nuclear architecture.

In contrast, H3K27me3 exhibited large-scale reorganization during transition from mESCs to EpiLCs and from EpiLCs to mPGCLCs. The H3K27me3 level was the highest in mESCs and decreased and was converted to a more flat, lawn-type distribution in EpiLCs. During induction of mPGCLCs from EpiLCs, the H3K27me 3 further decreased, reaching the lowest genome-wide level in $\mathrm{d} 2$ mPGCLCs. At this stage, however, H3K27me3 exhibited punctate enrichment around a set of genes, including genes for embryonic development such as Hox clusters. In accordance with the immunofluorescence analyses of mPGCs in vivo (Seki et al. 2005, 2007), the genome-wide $\mathrm{H} 3 \mathrm{~K} 27 \mathrm{me} 3$ level was then regained in $\mathrm{d} 6 \mathrm{mPGCLCs}$, with stronger enrichment around developmental regulators. These findings indicate that genes regulating embryonic development were more strongly repressed by PRC2 in mPGCLCs than in mESCs and EpiLCs.

In general, promoters show reciprocal distribution of repressive $\mathrm{H} 3 \mathrm{~K} 27 \mathrm{me} 3$ and active $\mathrm{H} 3 \mathrm{~K} 4 \mathrm{me} 3$ modifications, and the promoters with significant levels of both marks, the "bivalent" promoters, have been proposed to represent a poised transcriptional state (Voigt et al. 2013). During in vitro mPGC specification, EpiLCs exhibited the highest numbers of bivalent promoters, and such promoters were enriched in genes for developmental regulators, especially for neuron differentiation. Notably, such promoters in EpiLCs showed lower enrichment levels (i.e., closer to the threshold level of the definition of the enrichment) of both H3K27me3 and H3K4me3, suggesting that EpiLCs may represent a bona fide primed state for immediate differentiation into the three germ layers. The bivalent genes were reduced in number in d2 mPGCLCs because of up- and down-regulation of $\mathrm{H} 3 \mathrm{~K} 27 \mathrm{me} 3$ and $\mathrm{H} 3 \mathrm{~K} 4 \mathrm{me} 3$, respectively, suggesting that some of the developmental regulators may lose transcriptional potential in $\mathrm{d} 2 \mathrm{mPGCLCs}$. The numbers of the bivalent genes were then regained in $\mathrm{d} 6 \mathrm{mPGCLCs}$ with concomitant up-regulation of H3K27me3 and H3K4me3 levels, indicating that $\mathrm{d} 6 \mathrm{mPGCLCs}$ acquire a more permissive chromatin state around many developmental regulators with more strong PRC2-mediated repression.

The ChIP-seq analyses of the genome-wide binding sites for $\mathrm{T}$ and BLIMP1 revealed that in response to BMP4, T recruits $\mathrm{H} 3 \mathrm{~K} 27 \mathrm{ac}$ to activate a set of mesodermal genes as well as the mPGC determinants (Blimpl and Prdm14), and then BLIMP1 represses a broad range of developmental regulators, possibly by recruiting PRC2 and spreading H3K27me3. Collectively, the results of this study provided the first evidence for the dynamic 
remodeling of chromatin states for transcriptional regulation during germ cell specification and development, and demonstrated that T and BLIMP1 function successively to create active and repressive chromatin states, respectively, around their key targets for establishment of the germline epigenome.

\section{INDUCTION OF HUMAN PGCLCs}

The induction of human germ cell fate from human PSCs had been considered difficult, because unlike mPSCs, which bear a naïve pluripotency (Nichols and Smith 2009), hPSCs exhibit a primed pluripotency (Rossant 2008; Nichols and Smith 2009) and bear a property more similar to mEpiSCs (Brons et al. 2007; Tesar et al. 2007), which possess little, if any, potential to take on the germ cell fate (Hayashi and Surani 2009). Furthermore, there has been essentially no knowledge as to the mechanism for hPGC specification because of the difficulty in studying early human embryos (Fig. 1).

Recently, however, based on the concept of mPGCLC induction, two groups independently reported the induction of human PGC-like cells from hPSCs maintained on feeders (Irie et al. 2015) or in MEF-conditioned medium (Sugawa et al. 2015). In the study by Irie et al., SOX17 was shown to be critically required for induction of human PGC-like cells, and a "distinctive" pluripotency (suggested to correspond to the naïve pluripotency in mice) (Gafni et al. 2013) was shown to play a key role in the high competence of hESCs for the germ cell fate (Irie et al. 2015).

Sasaki et al. (2015) demonstrated the robust induction of human PGC-like cells from human iPSCs (hiPSCs) in a primed pluripotent state maintained under a defined, feeder-free condition. Sasaki et al. exploited hiPSCs bearing fluorescent reporters for BLIMP1 (BLIMP1-2AtdTomato: BT) and TFAP2C (TFAP2C-2A-EGFP: AG) and induced hiPSCs first into a state similar to the incipient mesoderm (incipient mesoderm-like cells: iMeLCs) by stimulation with activin A and a WNT-signaling agonist (CHIR99021) for $\sim 2$ d. iMeLCs showed expression of a number of early mesodermal markers such as EOMES, T, and MIXL1 at moderate levels and retained a high level of the key pluripotency factors POU5F1, $S O X 2$, and NANOG. When cultured as floating aggregates under conditions essentially identical to those for mPGCLC induction, iMeLCs exhibited robust $(\sim 30 \%-$ $40 \%$ ) activation of $\mathrm{BT}$ and $\mathrm{AG}$ in response to BMP4, and the BTAG-positive cells remained at least until day $8-10$ of induction.

The BTAG-positive cells exhibited a transcriptome similar to those of the gonadal PGCs of cynomolgus monkeys (roughly corresponding to E10.5-E13.5 mPGCs), except for the expression of late PGC genes such as Ddx4 and Piwil2, and were thus defined as cells representing early hPGCs (hPGCLCs). The hPGCLCs induced by Sasaki et al. (2015) also showed a transcriptome similar to those of human gonadal PGCs (except late PGC genes), and the TNAP/NANOS3-positive cells reported by Irie et al. (2015). The cells defined by Sugawa et al.
(2015), on the other hand, exhibited remarkably different gene expression profiles from those described by Sasaki et al. (2015) and Irie et al. (2015), presumably because of the differences in the induction and/or sorting strategies.

Notably, analyses of the dynamics of the transcriptome during hPGCLC induction revealed that hPGCLC specification occurred without the prominent activation of somatic programs. This is in contrast to the transient but eminent activation of the somatic mesodermal programthat is, the program for "pattern specification," "embryonic morphogenesis," and "gastrulation," etc., followed by robust repression during induction of mPGCLCs (Hayashi et al. 2011; Kurimoto et al. 2015). Instead, upon hPGCLC induction, the program related to "neuron differentiation" was transiently up-regulated and then repressed. Consistently, analyses of the hPGCLC induction from BLIMP1-knockout hiPSCs revealed that while BLIMP1 played critical roles in the specification and the maintenance of hPGCLCs (Irie et al. 2015; Sasaki et al. 2015), the role of BLIMP1 for the repression of somatic programs was variable (e.g., BLIMP1-deficient cells failed to maintain expression of $T$ and $M I X L 1$, whereas they failed to repress $E V X 1$ and $S P 5$, and had essentially no effects on EOMES and MSX2). On the other hand, BLIMP1 was critical to repress genes associated with "neuron differentiation" and to activate genes potentially important for hPGCs and pluripotency, such as NANOS3, $K L F 4, T F C P 2 L 1$, and TCL1B, indicating that BLIMP1 functions not only as a repressor but also as an activator for the transcriptional network for the hPGCLC fate. These findings highlight the difference in the molecular mechanisms of germ cell fate specification between humans and mice.

Collectively, the study by Sasaki et al. (2015) demonstrated that the human primed pluripotent state possesses robust competence for the germ cell fate and would require revision of the spectrum of developmental potential of human pluripotent states. Accordingly, gene expression analyses have suggested that hPSCs may bear a property somewhat between EpiLCs and EpiSCs.

\section{PERSPECTIVE}

In vitro reconstitution of mouse germ cell development from mPSCs provided a robust foundation for understanding the mechanism of germ cell development, including signaling pathways, transcriptional networks, and epigenetic regulation. There remain many open questions: for example, how and to what extent do the mPGCLCs undergo DNA methylation reprograming, and especially, what is the relationship between DNA methylation reprogramming and the histone modification states? Does the mechanism for DNA methylation reprogramming during mPGCLC induction resemble those during preimplantation development and/or during naïve mESC induction, as has been proposed previously (Seisenberger et al. 2012; Ficz et al. 2013; Leitch et al. 2013)? Is it possible to differentiate mPGCLCs into cells corresponding to mPGCs at late stages $(\sim \mathrm{E} 12.5)$ without the use of an aggregation culture with gonadal somatic cells 
and to induce mitotic arrest or meiosis by defined factors? Is it possible to differentiate mPGCLCs into SSCs or oocytes in vitro?

The mechanism for human germ cell development has largely been elusive because of inherent difficulties and/or limitations in the analysis of human embryos. The in vitro induction of hPGCLCs creates a unique opportunity for understanding the mechanism of human germ cell development, including the exploration of signaling pathways for and identification of the TFs essential for hPGC specification. On the other hand, for a more precise understanding of the mechanism of hPGC specification, it is critical to investigate the mechanism of PGC specification using a model organism that exhibits a mode of PGC specification similar to humans, such as nonhuman primates. Such investigations could clarify key issues such as the source for signaling molecules essential for PGC specification or the precise degree of similarity between the pathways for hPGCLC induction and hPGC specification in vivo.

Recently, three groups independently reported genome-wide DNA methylation and transcription profiles of hPGCs obtained from aborted embryos at the stages of arrival of the hPGCs to - and proliferation/differentiation within - the genital ridges (corresponding to $\sim$ E9.5-E13.5 in mice) (Gkountela et al. 2015; Guo et al. 2015; Tang et al. 2015), and revealed fundamental similarities in epigenome reprogramming between humans and mice. These discoveries have posed several critical challenges for the future. Namely, it will be important to explore whether hPGCLCs are further differentiated into cells corresponding to late-stage hPGCs in vitro, to examine the epigenetic reprogramming in such cells, and to determine whether such cells may have the potential to further differentiate into either spermatogonia or oocytes.

\section{ACKNOWLEDGMENTS}

We thank all of the laboratory members for their discussion and suggestions. The authors were supported in part by a Grant-in-Aid from the Ministry of Education, Culture, Sports, and Technology of Japan to K.K. (24681039), and by funds from Japan Science and Technology Agency Exploratory Research for Advanced Technology (JST-ERATO) to M.S.

\section{REFERENCES}

Aramaki S, Hayashi K, Kurimoto K, Ohta H, Yabuta Y, Iwanari H, Mochizuki Y, Hamakubo T, Kato Y, Shirahige K, et al. 2013. A mesodermal factor, T, specifies mouse germ cell fate by directly activating germline determinants. Dev Cell 27: 516-529.

Arand J, Wossidlo M, Lepikhov K, Peat JR, Reik W, Walter J. 2015. Selective impairment of methylation maintenance is the major cause of DNA methylation reprogramming in the early embryo. Epigenetics Chromatin 8: 1.

Brons IG, Smithers LE, Trotter MW, Rugg-Gunn P, Sun B, Chuva de Sousa Lopes SM, Howlett SK, Clarkson A, Ahrlund-Richter L, Pedersen RA, et al. 2007. Derivation of plu- ripotent epiblast stem cells from mammalian embryos. Nature 448: $191-195$.

Daley GQ. 2007. Gametes from embryonic stem cells: A cup half empty or half full? Science 316: 409-410.

Dawlaty MM, Ganz K, Powell BE, Hu YC, Markoulaki S, Cheng AW, Gao Q, Kim J, Choi SW, Page DC, et al. 2011. Tet1 is dispensable for maintaining pluripotency and its loss is compatible with embryonic and postnatal development. Cell Stem Cell 9: 166-175.

Ficz G, Hore TA, Santos F, Lee HJ, Dean W, Arand J, Krueger F, Oxley D, Paul YL, Walter J, et al. 2013. FGF signaling inhibition in ESCs drives rapid genome-wide demethylation to the epigenetic ground state of pluripotency. Cell Stem Cell 13: $351-359$.

Gafni O, Weinberger L, Mansour AA, Manor YS, Chomsky E, Ben-Yosef D, Kalma Y, Viukov S, Maza I, Zviran A, et al. 2013. Derivation of novel human ground state naive pluripotent stem cells. Nature 504: 282-286.

Ginsburg M, Snow MH, McLaren A. 1990. Primordial germ cells in the mouse embryo during gastrulation. Development 110: $521-528$.

Gkountela S, Zhang KX, Shafiq TA, Liao WW, Hargan-Calvopina J, Chen PY, Clark AT. 2015. DNA demethylation dynamics in the human prenatal germline. Cell 161: 1425-1436.

Guo JU, Su Y, Zhong C, Ming GL, Song H. 2011. Hydroxylation of 5-methylcytosine by TET1 promotes active DNA demethylation in the adult brain. Cell 145: 423-434.

Guo F, Yan L, Guo H, Li L, Hu B, Zhao Y, Yong J, Hu Y, Wang $\mathrm{X}$, Wei Y, et al. 2015. The transcriptome and DNA methylome landscapes of human primordial germ cells. Cell 161: $1437-1452$.

Hajkova P, Erhardt S, Lane N, Haaf T, El-Maarri O, Reik W, Walter J, Surani MA. 2002. Epigenetic reprogramming in mouse primordial germ cells. Mech Dev 117: 15-23.

Hajkova P, Jeffries SJ, Lee C, Miller N, Jackson SP, Surani MA. 2010. Genome-wide reprogramming in the mouse germ line entails the base excision repair pathway. Science 329: $78-82$.

Hayashi K, Surani MA. 2009. Self-renewing epiblast stem cells exhibit continual delineation of germ cells with epigenetic reprogramming in vitro. Development 136: 3549-3556.

Hayashi K, Ohta H, Kurimoto K, Aramaki S, Saitou M. 2011. Reconstitution of the mouse germ cell specification pathway in culture by pluripotent stem cells. Cell 146: 519-532.

Hayashi K, Ogushi S, Kurimoto K, Shimamoto S, Ohta H, Saitou M. 2012. Offspring from oocytes derived from in vitro primordial germ cell-like cells in mice. Science 338: $971-$ 975.

Heard E, Martienssen RA. 2014. Transgenerational epigenetic inheritance: Myths and mechanisms. Cell 157: 95-109.

Herrmann BG, Labeit S, Poustka A, King TR, Lehrach H. 1990. Cloning of the $\mathrm{T}$ gene required in mesoderm formation in the mouse. Nature 343: 617-622.

Irie N, Weinberger L, Tang WW, Kobayashi T, Viukov S, Manor YS, Dietmann S, Hanna JH, Surani MA. 2015. SOX17 is a critical specifier of human primordial germ cell fate. Cell 160: 253-268.

Kagiwada S, Kurimoto K, Hirota T, Yamaji M, Saitou M. 2013. Replication-coupled passive DNA demethylation for the erasure of genome imprints in mice. EMBO J 32: 340-353.

Kaufman MH. 1992. The atlas of mouse development. Academic Press, London.

Kriaucionis S, Heintz N. 2009. The nuclear DNA base 5hydroxymethylcytosine is present in Purkinje neurons and the brain. Science 324: 929-930.

Kurimoto K, Yabuta Y, Ohinata Y, Ono Y, Uno KD, Yamada RG, Ueda HR, Saitou M. 2006. An improved single-cell cDNA amplification method for efficient high-density oligonucleotide microarray analysis. Nucleic Acids Res 34: e42.

Kurimoto K, Yabuta Y, Ohinata Y, Shigeta M, Yamanaka K, Saitou M. 2008. Complex genome-wide transcription dynamics orchestrated by Blimp1 for the specification of the germ cell lineage in mice. Genes Dev 22: 1617-1635. 
Kurimoto K, Yabuta Y, Hayashi K, Ohta H, Kiyonari H, Mitani T, Moritoki Y, Kohri K, Kimura H, Yamamoto T, et al. 2015. Quantitative dynamics of chromatin remodeling during germ cell specification from mouse embryonic stem cells. Cell Stem Cell 16: 517-532.

Lawson KA, Dunn NR, Roelen BA, Zeinstra LM, Davis AM, Wright CV, Korving JP, Hogan BL. 1999. Bmp4 is required for the generation of primordial germ cells in the mouse embryo. Genes Dev 13: 424-436.

Lee HJ, Hore TA, Reik W. 2014. Reprogramming the methylome: Erasing memory and creating diversity. Cell Stem Cell 14: 710-719.

Leitch HG, McEwen KR, Turp A, Encheva V, Carroll T, Grabole N, Mansfield W, Nashun B, Knezovich JG, Smith A, et al. 2013. Naïve pluripotency is associated with global DNA hypomethylation. Nat Struct Mol Biol 20: 311-316.

Liu P, Wakamiya M, Shea MJ, Albrecht U, Behringer RR, Bradley A. 1999. Requirement for Wnt3 in vertebrate axis formation. Nat Genet 22: 361-365.

Luckett WP. 1978. Origin and differentiation of the yolk sac and extraembryonic mesoderm in presomite human and rhesus monkey embryos. Am J Anat 152: 59-97.

Ma Z, Swigut T, Valouev A, Rada-Iglesias A, Wysocka J. 2011. Sequence-specific regulator Prdm14 safeguards mouse ESCs from entering extraembryonic endoderm fates. Nat Struct Mol Biol 18: $120-127$.

Magnusdottir E, Dietmann S, Murakami K, Gunesdogan U, Tang F, Bao S, Diamanti E, Lao K, Gottgens B, Azim Surani M. 2013. A tripartite transcription factor network regulates primordial germ cell specification in mice. Nat Cell Biol 15: 905-915.

Matsui Y, Zsebo K, Hogan BL. 1992. Derivation of pluripotential embryonic stem cells from murine primordial germ cells in culture. Cell 70: $841-847$.

Nakaki F, Hayashi K, Ohta H, Kurimoto K, Yabuta Y, Saitou M. 2013. Induction of mouse germ-cell fate by transcription factors in vitro. Nature 501: 222-226.

Nichols J, Smith A. 2009. Naive and primed pluripotent states. Cell Stem Cell 4: 487-492.

Ohinata Y, Payer B, O'Carroll D, Ancelin K, Ono Y, Sano M, Barton SC, Obukhanych T, Nussenzweig M, Tarakhovsky A, et al. 2005. Blimp1 is a critical determinant of the germ cell lineage in mice. Nature 436: 207-213.

Ohinata Y, Ohta H, Shigeta M, Yamanaka K, Wakayama T, Saitou M. 2009. A signaling principle for the specification of the germ cell lineage in mice. Cell 137: 571-584.

Popp C, Dean W, Feng S, Cokus SJ, Andrews S, Pellegrini M, Jacobsen SE, Reik W. 2010. Genome-wide erasure of DNA methylation in mouse primordial germ cells is affected by AID deficiency. Nature 463: 1101-1105.

Rivera-Perez JA, Magnuson T. 2005. Primitive streak formation in mice is preceded by localized activation of Brachyury and Wnt3. Dev Biol 288: 363-371.

Rossant J. 2008. Stem cells and early lineage development. Cell 132: 527-531.

Saitou M, Yamaji M. 2012. Primordial germ cells in mice. Cold Spring Harb Perspect Biol 4: a008375.

Saitou M, Barton SC, Surani MA. 2002. A molecular programme for the specification of germ cell fate in mice. Nature 418: $293-300$.

Saitou M, Kagiwada S, Kurimoto K. 2012. Epigenetic reprogramming in mouse pre-implantation development and primordial germ cells. Development 139: 15-31.

Sasaki K, Yokobayashi S, Nakamura T, Okamoto I, Yabuta Y, Kurimoto K, Ohta H, Moritoki Y, Iwatani C, Tsuchiya H, et al. 2015. Robust in vitro induction of human germ cell fate from pluripotent stem cells. Cell Stem Cell 17: 178-194.

Seisenberger S, Andrews S, Krueger F, Arand J, Walter J, Santos F, Popp C, Thienpont B, Dean W, Reik W. 2012. The dynamics of genome-wide DNA methylation reprogramming in mouse primordial germ cells. Mol Cell 48: 849-862.
Seki Y, Hayashi K, Itoh K, Mizugaki M, Saitou M, Matsui Y. 2005. Extensive and orderly reprogramming of genome-wide chromatin modifications associated with specification and early development of germ cells in mice. Dev Biol 278: $440-458$.

Seki Y, Yamaji M, Yabuta Y, Sano M, Shigeta M, Matsui Y, Saga Y, Tachibana M, Shinkai Y, Saitou M. 2007. Cellular dynamics associated with the genome-wide epigenetic reprogramming in migrating primordial germ cells in mice. Development 134: 2627-2638.

Sharif J, Muto M, Takebayashi S, Suetake I, Iwamatsu A, Endo TA, Shinga J, Mizutani-Koseki Y, Toyoda T, Okamura K, et al. 2007. The SRA protein Np95 mediates epigenetic inheritance by recruiting Dnmt1 to methylated DNA. Nature 450: $908-912$.

Sugawa F, Arauzo-Bravo MJ, Yoon J, Kim KP, Aramaki S, Wu G, Stehling M, Psathaki OE, Hubner K, Scholer HR. 2015. Human primordial germ cell commitment in vitro associates with a unique PRDM14 expression profile. EMBO J 34: $1009-1024$.

Surani MA, Hajkova P. 2010. Epigenetic reprogramming of mouse germ cells toward totipotency. Cold Spring Harb Symp Quant Biol 75: 211-218.

Tahiliani M, Koh KP, Shen Y, Pastor WA, Bandukwala H, Brudno Y, Agarwal S, Iyer LM, Liu DR, Aravind L, et al. 2009. Conversion of 5-methylcytosine to 5-hydroxymethylcytosine in mammalian DNA by MLL partner TET1. Science 324: $930-935$.

Tanaka SS, Nakane A, Yamaguchi YL, Terabayashi T, Abe T, Nakao K, Asashima M, Steiner KA, Tam PP, Nishinakamura R. 2013. Dullard/Ctdnep1 modulates WNT signalling activity for the formation of primordial germ cells in the mouse embryo. PLoS One 8: e57428.

Tang WW, Dietmann S, Irie N, Leitch HG, Floros VI, Bradshaw CR, Hackett JA, Chinnery PF, Surani MA. 2015. A unique gene regulatory network resets the human germline epigenome for development. Cell 161: 1453-1467.

Tesar PJ, Chenoweth JG, Brook FA, Davies TJ, Evans EP, Mack DL, Gardner RL, McKay RD. 2007. New cell lines from mouse epiblast share defining features with human embryonic stem cells. Nature 448: $196-199$.

Vincent SD, Dunn NR, Sciammas R, Shapiro-Shalef M, Davis MM, Calame K, Bikoff EK, Robertson EJ. 2005. The zinc finger transcriptional repressor Blimp1/Prdm1 is dispensable for early axis formation but is required for specification of primordial germ cells in the mouse. Development 132: 13151325.

Voigt P, Tee WW, Reinberg D. 2013. A double take on bivalent promoters. Genes Dev 27: 1318-1338.

Yabuta Y, Kurimoto K, Ohinata Y, Seki Y, Saitou M. 2006. Gene expression dynamics during germline specification in mice identified by quantitative single-cell gene expression profiling. Biol Reprod 75: 705-716.

Yamaguchi S, Hong K, Liu R, Shen L, Inoue A, Diep D, Zhang K, Zhang Y. 2012. Tet1 controls meiosis by regulating meiotic gene expression. Nature 492: 443-447.

Yamaguchi S, Shen L, Liu Y, Sendler D, Zhang Y. 2013. Role of Tet1 in erasure of genomic imprinting. Nature 504: 460464.

Yamaji M, Seki Y, Kurimoto K, Yabuta Y, Yuasa M, Shigeta M, Yamanaka K, Ohinata Y, Saitou M. 2008. Critical function of Prdm14 for the establishment of the germ cell lineage in mice. Nat Genet 40: 1016-1022.

Yamaji M, Ueda J, Hayashi K, Ohta H, Yabuta Y, Kurimoto K, Nakato R, Yamada Y, Shirahige K, Saitou M. 2013. PRDM14 ensures naive pluripotency through dual regulation of signaling and epigenetic pathways in mouse embryonic stem cells. Cell Stem Cell 12: 1-15.

Ying QL, Wray J, Nichols J, Batlle-Morera L, Doble B, Woodgett J, Cohen P, Smith A. 2008. The ground state of embryonic stem cell self-renewal. Nature 453: 519-523. 


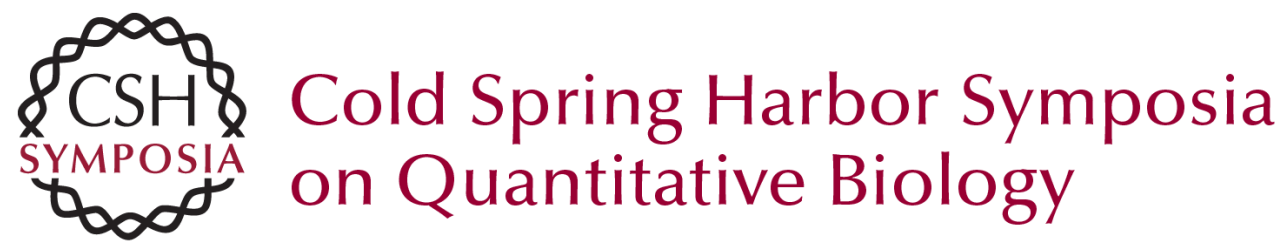

\section{Mechanism and Reconstitution In Vitro of Germ Cell Development in Mammals}

Kazuki Kurimoto and Mitinori Saitou

Cold Spring Harb Symp Quant Biol 2015 80: 147-154 originally published online December 7, 2015

Access the most recent version at doi:10.1101/sqb.2015.80.027425

References This article cites 61 articles, 17 of which can be accessed free at: http://symposium.cshlp.org/content/80/147.full.html\#ref-list-1

\section{License}

Email Alerting Receive free email alerts when new articles cite this article - sign up in Service the box at the top right corner of the article or click here. 